\title{
Stochastic Modeling of Streamer Transitions
}

\author{
M. Kim ${ }^{1}$, B. Murphy ${ }^{2}$, and R.E. Hebner ${ }^{2}$ \\ ${ }^{1}$ ASML, 77 Danbury Road, Wilton, CT 06897 USA \\ ${ }^{2}$ University of Texas at Austin, 1 University Station, \#R7000, Austin, TX 78712 USA
}

\begin{abstract}
Simulations of the growth of streamers using a model based on the stochastic growth of a branching fractal tree have shown good fidelity in matching the morphology of streamers in liquids. An important aspect that has not yet been simulated is the transition between Type 1 and Type 2 streamers. This work explores the effect of a variation of the applied voltage during streamer growth as well as the effect of differing amounts of available electrons during growth. Neither of these assumptions produced the experimentally observed transitions. These results produced a possible approach that could modify the step length in the simulation in relation to the electric field that may trigger the transition. It also points out that the transition might be due to hydrodynamic instabilities that are not captured by the model.
\end{abstract}

\section{INTRODUCTION}

Earlier work by Fowler, Davaney, and Hagedorn [1] showed that the morphology of an anode streamer could be modeled as stochastic growth of a branching fractal tree in point-plane geometry. Because one of the concerns about the earlier work is that the electric field dependence appeared to be unphysical, Kim, Hebner, and Hallock [2] refined the model so that it operates under assumptions that are consistent with those that have proven useful in earlier investigations. Specifically, linear electric field dependence was assumed and there is an assumed variability in the number density of available electrons. Computations using this assumption also produce the same range of morphologies that has been measured in experiments.

This investigation explores the feasibility of extending this model to the description of the transition between Type 1 and Type 2 streamers [3]. The key morphology difference between these two streamer types is that Type 1 streamers are "bushy" and spherical to hemispherical. Type 2 streamers are sparse and much more linear in growth pattern. While significant progress has been made in modeling streamer propagation $[2,4,5]$, there have been no successful explanations of this transition.

A challenge in modeling this transition is that it is unknown if the transition is caused by a stochastic change in a single process during streamer propagation or if the electric field growth leads to a regime in which a different physical process dominates. This work focuses on exploring the first and simpler option, i.e., that there is but a single process.

Conceptually, there are three key assumptions used in this model:

1. The growth is driven by the electric field to the first power.

2. The growth is stochastic.

3. Each growth step is of limited length.
In this model, as applied in earlier work, the only influence of the electric field was that it had to be above a threshold level. The basic concept was that for fields that were too small, electrons in the fluid would not acquire sufficient energy from the electric field to sustain a breakdown. Once the threshold was passed, further strengthening of the field was assumed to affect things like emitted light intensity and the conductivity of an individual streamer branch, but not the future steps in the growth. The stochastic nature of the growth is assumed to be attributable to the stochastic nature of available electrons. An available electron is one that through some random process, e.g., cosmic radiation, scattering, etc., is in a position to be accelerated by the electric field and gain sufficient energy to produce electron impact ionization [6].

The growth is, in this and related work [2,7], typically assumed to be one or a few grid steps from the end of a previously growing streamer. After each growth step, the new electric field distribution is calculated and a new growth step is initiated.

As shown in [1], [2], [4], [6], and [7], this simulation approach has been effective in replicating key aspects of the morphology of streamers in liquids. Although the models have predominantly been focused on the behavior of streamers originating from an anode, the same basic streamer morphology is seen in streamers whether they originate from an anode or a cathode. So, the computational approach should be applicable to streamers of either polarity, at least to the level that they have been validated so far. An interesting difference between the two types of streamers, however, is in the electron source. For streamers originating at the anode, it was assumed that the electrons originated from natural processes in the liquid under the non-equilibrium effect of a strong electric field. For cathode streamers, the same process may occur, although the active volumes may be different. In addition, there is a second plausible process, as electrons have long been postulated as being emitted from the tip of a growing cathode streamer [8]. These electrons would be accelerated by the field to cause streamer growth.

The existing modeling attempts have not been able to stimulate the transition using this stochastic approach. The work reported here shows the effect of two different assumptions. First, in many of the experiments showing a clear transition, the applied voltage was increasing during streamer growth. Simulations are reported showing the effect of only voltage increase during growth. Second, the effect of modifying the number of electrons available to augment growth is simulated. 


\section{EXPERIMENTAL BACKGROUND}

There are four sets of experimental observations that guided this investigation. The most fundamental is that for streamers originating at both the anode and the cathode, there can be a transition from Type 1 to Type 2 streamer morphology [3]. This implies that such a transition is a fundamental feature of breakdown and should be a natural consequence of any simulation of the process.

The second set of experiments [9] involved adding a particulate conductor, carbon black, to a liquid dielectric and recording the effect of the material on the behavior of the streamer. This experiment showed that the addition of the particulate conductor did not have any significant effect on the velocity of either Type 1 or Type 2 streamers. It did, however, affect the transition between the two. Specifically, as the density of carbon black increased, the transition between Type 1 and Type 2 occurred closer to the electrode from which it initiated. The earlier mode shift led to a faster breakdown as Type 2 streamers propagate faster than Type 1 streamers.

The third set of experiments involved the addition of a chemical additive to the test liquid [10]. In these studies, the streamer initiated from a point anode. The first effect of this additive was to produce growth of Type 1 streamers across a significant portion of the inter-electrode gap. In this sense, the anode streamer looked like a typical cathode streamer.

The final set of observations was the electro-optical measurement of the dynamic electric field preceding a streamer [11]. These experimental measurements show that the electric field in the region between a streamer and the electrode toward which it is propagating can be computed assuming the streamer is a conductor. This is a useful result because the resistivity of individual channels varies with time [12]. This experimental result makes it much easier to compute the electric field ahead of the streamer by showing it is not necessary to account for the variability in each channel. Obviously, at some point very close to the streamer, one would expect this result to fail, but that is presumed to be smaller than the regions of interest for the streamer propagation process.

\section{SimulATIONS}

The purpose of the simulations was to explore the sensitivity of growth to various assumptions. The first investigation was to let the voltage increase while the streamer propagated across the inter-electrode gap. This situation was investigated because many of the experimental investigations used pulsed voltages that varied by this amount. It was important to determine if this variability would produce the transition from Type 1 to Type 2 streamers.

It was expected that this variation would not lead to the observed transitions, as the change in field strength between the streamer and the electrode toward which it is propagating is larger than this due to the dynamic geometry of growth. This expectation was confirmed. Fig. 1 shows cases in which the field was held constant during growth or grew by $10 \%$,
$20 \%$, and $50 \%$ and no transition was observed nor was there any apparent difference in growth dynamics.

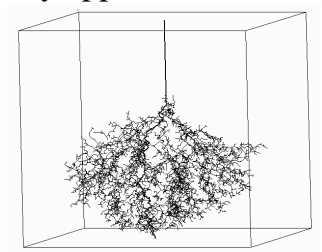

a

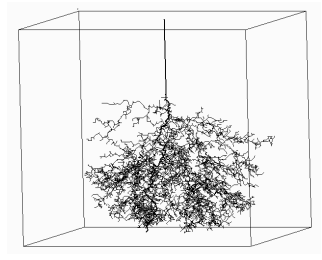

c

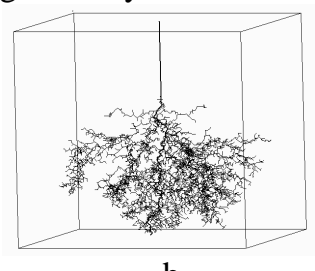

b

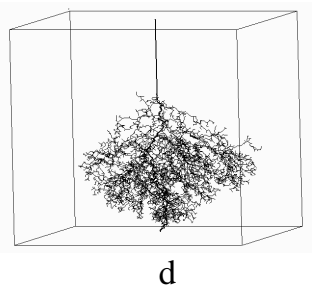

Fig. 1. Simulations of streamer growth with a) the applied voltage staying constant during streamer growth and the applied voltage growing by b) $10 \%$, c) $20 \%$, and d) $50 \%$ during growth

A second simulation varied the number of available electrons as the streamer grew. Fig. 2 and 3 summarize this information. Specifically, Fig. 2 shows a streamer simulation in which the electron density is kept constant. Fig. 3 shows a situation in which the presumed electron density is allowed to increase by a factor of a) 50, b) 20, c) 10 and in d) decrease by a factor of two as the streamer propagates. As expected from previous work, increasing the number of available electrons increases the streamer density but does not simulate transition.

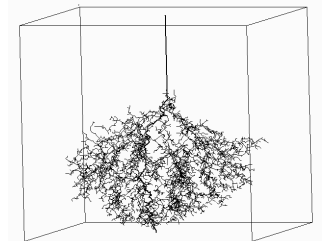

Fig. 2. Streamer growth with presumed constant density of available electrons

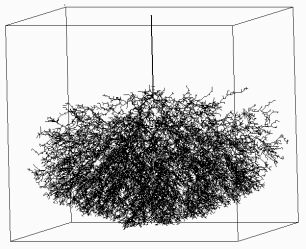

a

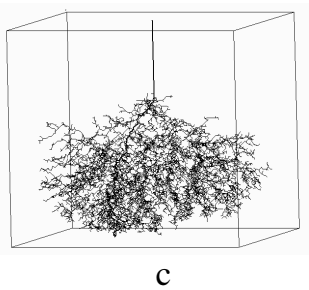

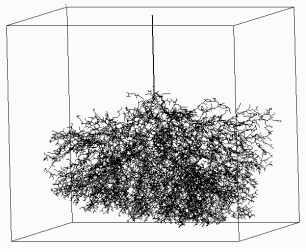

$\mathrm{b}$

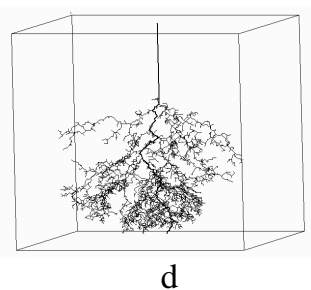

Fig. 3. Streamer growth in which the presumed electron density is allowed to increase by a factor of a) 50, b) 20 , c) 10 and in d) decrease by a factor of two as the streamer propagates

Following this work, an additional simulation was done. This simulation looked at the variation of the electric field as 
the streamer propagated. An example of this simulation is shown in Fig. 4.
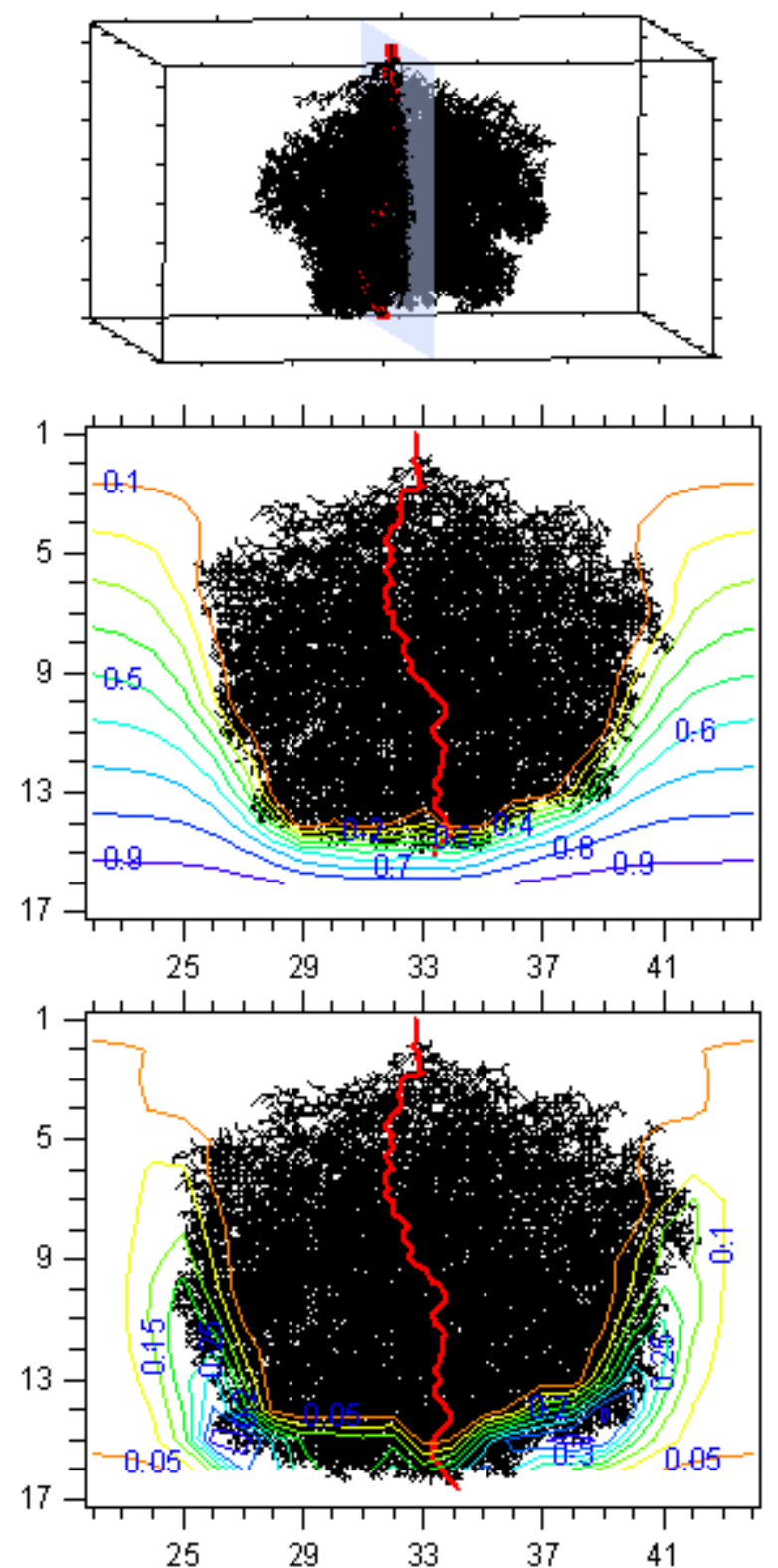

Fig. 4. The upper computer graphic depicts the growth of a dense streamer (Type 1), the middle graphic shows equipotential contours, and the lower graphic shows the intensification of the electric field due to the growing streamer

\section{DISCUSSION}

One clear result from this investigation is that the simulation assumptions were no more effective at producing the transitions than were the assumptions used in an earlier investigation [2]. This raises the possibility that the simulation approach is not sufficiently robust to simulate such a transition, but does not yet prove such an assertion. The investigations suggest that there may be an approach that would demonstrate a transition. Assessments like those in Fig. 4 , suggest that as the streamer grows, there is an enhancement of the electric field at the leading edge of the streamer. This suggests a different approach to simulation. In earlier work, the size of a growth step was chosen a priori. The next investigation will have the length of a step be driven by the strength of the electric field. This approach would allow a much longer step as a Type 1 streamer grows, possibly triggering a transition to a Type 2 streamer.

There is an analysis, however, that raises the possibility that the stochastic modeling approach used in this work may not include sufficient physics to predict the transition. Kupershtokh and Medvedev [13] have suggested that a strong electric field in a liquid triggers an instability that results in a two phase system of vapor filaments in a liquid. The filaments are aligned with the field. This raises the possibility that the transition could result from a hydrodynamic instability rather than be triggered by the effect of the electric field on available electrons.

\section{REFERENCES}

[1] H.A. Fowler, J.E. Devaney, and J.G. Hagedorn, "Growth model for filamentary streamers in an ambient field," IEEE Trans. on Dielectr. Electr. Insul., vol. 10, pp. 73-79, 2003.

[2] M. Kim; R.E. Hebner, G.A. Hallock, "Modeling the growth of streamers during liquid breakdown," IEEE Trans. on Dielectr. and Electrical Insulation, vol. 15, pp.547-553, 2008.

[3] R.E. Hebner, "Measurement of electrical breakdown in liquids," Proc. NATO Advanced Study Institute on the Liquid State and its Electrical Properties, Sintra, Portugal, Plenum Pub. Corp., New York City, NY, pp. $519-537,1987$.

[4] A.L. Kuperstokh, et al., "Stochastic model of breakdown initiation in dielectric liquids," J. Phys. D: Appl. Phys, vol. 35, pp. 3106-3121, 2002.

[5] F. O'Sullivan, et al., "A model for the initiation and propagation of positive streamers in transformer oil," Proc. IEEE International Symposium on Electrical Insulation, 2008, in press.

[6] M. Kim and R.E. Hebner, "Initiation from a point anode in a dielectric liquid," IEEE Trans. Dielectr. Electr. Insul., vol. 13, pp. 1254-1260, 2006.

[7] A.L. Kupershtokh, V. Charalambskos, D. Agoris, and D.I. Karpov, "Simulation of breakdown in air using cellular automata with streamer to leader transition," J. Phys. D: Appl. Phys., vol. 34, pp. 936-946, 2001.

[8] R.E. Hebner, E.F. Kelley, E.O. Forster, and G.J. FitzPatrick, "Observation of prebreakdown and breakdown phenomena in liquid Hydrocarbons II. non-uniform field conditions," IEEE Trans. Electr. Insul., vol. EI-20, pp. 281-292, 1985.

[9] R.E. Hebner, E.F. Kelley, G.J. Fitzpatrick, E.U. Forster, "The effect of impurities on positive streamer propagation in n-Hexane," Proc. Conf. on Elec. Insul. Dielectric Phen. (CEIDP), pp. 26-34, 1983.

[10] R.E. Hebner, "Factors contributing to streamer morphology," Proc. 14th Int. Conf. on Diel. Liquids, pp.155-158, 2002.

[11] E. F. Kelley and R. E. Hebner, "The electric field distribution associated with prebreakdown phenomena in nitrobenzene," J. Appl. Phys., vol. 52, pp. 191-196, 1981.

[12] A.L. Kupershtokh and D.I. Karpov, "Simulation of the development of branching streamer structures in dielectrics liquids with pulsed conductivity of channels," Tech. Phys. Lett., vol. 32, pp.406-409, 2006.

[13] A.L. Kupershtokh and D.A. Medvedev, "Anisotropic instability of a dielectric liquid in a strong uniform electric field: decay into a twophase system of vapor filaments in a liquid," Phys. Rev. E, vol. 74, pp. 021505-1 to 021505-5, 2006. 